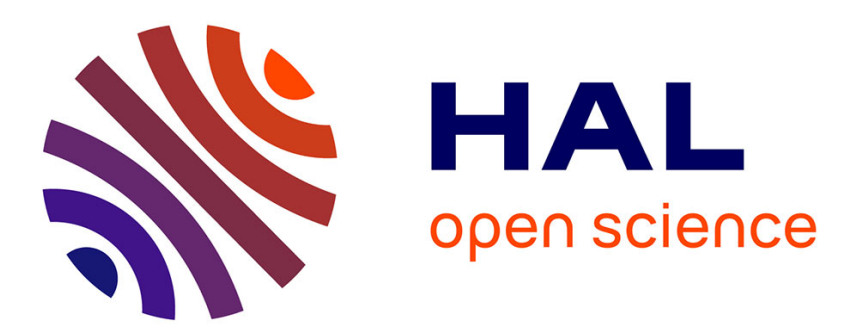

\title{
DÉTECTION DE FRÉQUENCE ET DE PHASE EN CONTRÔLE ACTIF SYNCHRONE
}

\author{
M. de Coligny, A. Douerin
}

\section{To cite this version:}

M. de Coligny, A. Douerin. DÉTECTION DE FRÉQUENCE ET DE PHASE EN CONTRÔLE ACTIF SYNCHRONE. Journal de Physique IV Proceedings, 1992, 02 (C1), pp.C1-523-C1-526. 10.1051/jp4:19921113 . jpa-00251068

\section{HAL Id: jpa-00251068 https://hal.science/jpa-00251068}

Submitted on 1 Jan 1992

HAL is a multi-disciplinary open access archive for the deposit and dissemination of scientific research documents, whether they are published or not. The documents may come from teaching and research institutions in France or abroad, or from public or private research centers.
L'archive ouverte pluridisciplinaire HAL, est destinée au dépôt et à la diffusion de documents scientifiques de niveau recherche, publiés ou non, émanant des établissements d'enseignement et de recherche français ou étrangers, des laboratoires publics ou privés. 


\title{
DÉTECTION DE FREQUENCE ET DE PHASE EN CONTRÔLE ACTIF SYNCHRONE
}

\author{
M. de COLIGNY et A. DOUERIN \\ Laboratoire d'Etudes Aérodynamiques, Bâtiment H, 40 avenue du Recteur Pineau, F-86022 Poitiers \\ cedex, France
}

\begin{abstract}
Time domain methods for synchronous active control noise need very precise measurement of frequency and phase. In most cases an error detector is put at the place where attenuation is required, and phase detection is achieved by optical or mechanical device. In order to suppress this external mechanical sensor, we use an algorithm calculating the frequency and phase, from main signal given by a pressure sensor or accelerometer. The method is derived from digital phase locked loops, specially using zero-crossing estimation by a least mean square method with specific noise-suppressing filter. Real-time computation is implemented on a TMS 320C25 signal processing system board. Trials demonstrate the reliability of such frequency detection in practical environment with perturbations.
\end{abstract}

\section{INTRODUCTION}

Dans tout système synchrone, la connaissance de la fréquence du signal est par définition, nécessaire: en particulier, dans les systèmes de contrôle actif synchrone d'ondes acoustiques, de vibrations ou de pulsations d'écoulement. De plus la fréquence est une des trois grandeurs caractéristiques d'un signal périodique avec l'amplitude et la phase. La détection de cette information est le plus souvent réalisée à l'aide d'un codeur optique, d'un tachymètre, ou tout autre système mécanique. La mise en place de codeurs sur sites industriels pose de tels problèmes qu'il est souhaitable de s'affranchir de leur présence pour de simples raisons mécaniques par exemple.

Dans le domaine de l'acoustique, le contrôle actif de bruit résulte d'une interférence entre le champ de la source de bruit (source primaire) et celui produit à l'aide d'un contrôleur (source secondaire) [1]. La plupart de ces systèmes utilise un algorithme temporel de minimisation de type LMS avec référence filtrée. Dans le cas synchrone les signaux traités sont quasi périodiques. Le signal de référence pris à partir de la source primaire doit être connecté au contrôleur dans le but d'avoir un signal de synchronisation cohérent avec la source primaire. Ce signal de synchronisation est donc obtenu par l'intermédiaire d'un système externe. Dans [2] par exemple, le signal de référence est obtenu à partir d'un codeur dans le cas de machine tournante comme source primaire. Dans le domaine des vibrations, il s'agit de contrôler directement la vibration elle même à l'aide d'actuateur (pot vibrant, céramique piézo-électrique) [3]. Enfin dans le cas de contrôle actif de pulsation de débit [4], [5] développé au LEA et qui consiste à placer dans l'écoulement une perte de charge singulière mobile, l'information de fréquence est encore obtenue à l'aide d'un codeur optique. La mesure des grandeurs fluctuantes est permise grâce à un échantillonnage conditionnel, c'est à dire lorsque l'horloge d'acquisition est 'conditionnée' sur le phénomène physique.

Afin de remplacer le mode de détection externe (codeur) par une information obtenue à partir d'un signal représentatif du phénomène issu d'un accéléromètre, d'un capteur de pression ou d'un microphone..., nous avons mis au point une procédure algorithmique de détermination directe de fréquence. La méthode utilisée est basée sur le principe de fonctionnement des boucles numériques à verrouillage de phase (DPLL), et plus particulièrement celles utilisant la détection de "zéro" [6]. Les boucles à détection de zéro consistent à estimer la phase d'un signal donné par recherche du franchissement du signal par zéro. Ce choix, plutôt que celui d'une boucle analogique, vient du fait que ce type de boucles (DPLL) est mieux adapté à un fonctionnement en basse fréquence $(<100 \mathrm{~Hz})$ que leurs équivalentes analogiques (problèmes dus au choix et au bon fonctionnement des composants en 
basse fréquence). De plus, elles permettent facilement un traitement en temps réel de signaux échantillonnés : par exemple pour une correction à avance de phase numérique.

DETECTEUR DE FREQUENCE

\section{Constitution générale}

On suppose que le signal à traiter est composé d'harmoniques et d'un fondamental de fréquence fo, fréquence que l'on cherche à estimer. Il s'agit en fait de faire une estimation des instants $t_{0} i$ correspondant aux passages par zéro, l'estimation de la fréquence fondamentale s'effectuant directement en posant $f_{o}=1 / T$ avec $T=t_{o}{ }^{i}-t_{o}{ }^{i-2}$. Ce détecteur de fréquence est donc constitué essentiellement d'un échantillonneur, d'un filtre passe-bas à phase linéaire, d'un détecteur de zéro et d'un estimateur des $t_{0} \mathfrak{i}$. Le détecteur de fréquence présenté ici fonctionne donc selon le schéma suivant:

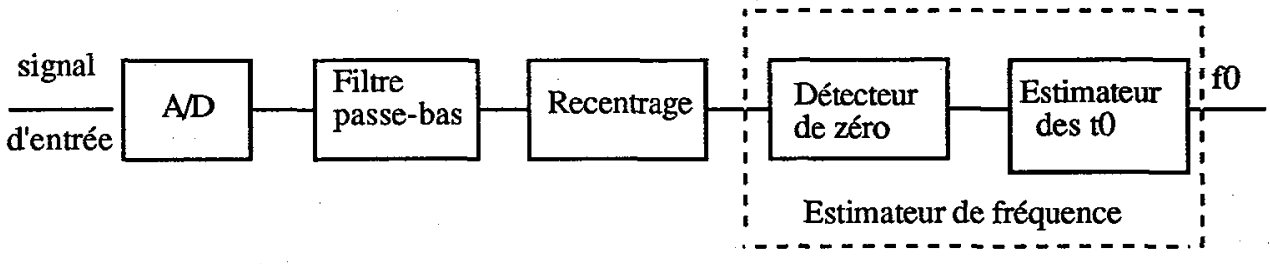

\section{Principe de fonctionnement}

Le signal d'entrée que l'on note $\mathrm{P}(\mathrm{t})$ est échantillonné à la fréquence $\mathrm{fe}=1 / \mathrm{Te}$. Ainsi à l'instant $\mathrm{t}_{\mathrm{k}}$ le signal d'entrée s'écrit :

$$
P\left(t_{k}\right)=P(k T e)=P(k)
$$

Ce signal $P_{k}$ est filtré numériquement. Nous avons choisi pour réaliser ce détecteur d'utiliser un filtre passe-bas de type RIF [7], [8]. En effet, ce filtre possède l'avantage d'être toujours stable et d'être facile à réaliser . Ce filtre sert donc à éliminer les harmoniques afin de lever toute ambiguité sur les

franchissements par zéro. Ce signal est recentré par soustraction à chaque échantillon de $\overline{\mathbf{P}}$ la valeur moyenne du signal d'entrée. Le signal filtré recentré est noté :

$$
P^{*}(k)=F[P(k)]-\bar{P}
$$

On peut alors considérer que le signal à l'entrée du détecteur de zéro est quasi sinusoïdal.

a) Détecteur de zéro:

Le détecteur de zéro a en réalité deux fonctions. Comme son nom l'indique, il sert en premier lieu à détecter les passages par zéro, c'est à dire, les instants où la sinusoïde, à son entrée, coupe l'axe des abscisses. Outre, sa fonction première, c'est lui qui donne l'ordre à l'estimateur de démarrer son calcul d'estimation et lui transfert les données nécessaires.

Le signal de commande:

Il s'agit pour le détecteur de zéro d'envoyer un signal, par exemple une impulsion, à l'estimateur à chaque passage par zéro du signal d'entrée P*. Ainsi, le signal de commande doit avoir les caractéristiques suivantes:

$$
\begin{array}{rlrl}
c(k) & =1 & \text { si passage par zéro } & \text { soit } P^{*}=\sin \left(2 \pi f_{0} t\right) \\
& =0 & \text { sinon }
\end{array}
$$

La détection de zéro se fait simplement en étudiant le signe du produit :

$$
\mathrm{P} *_{\mathrm{i}} \mathrm{P} *_{\mathrm{i}-1}
$$

Compte tenu de (C1) et (C2), le signal de commande doit donc satisfaire aux conditions suivantes:

$c(k)=\operatorname{sgn}\left[1-\operatorname{sgn}\left(P^{*}{ }_{k}{ }^{*} k-1\right)\right]$ 
Les registres de transfert:

Ces registres sont au nombre de deux. Le premier contient les derniers échantillons acquis. Nous nous sommes limités à ne conserver que les cinq derniers : ce nombre étant apparu suffisant au fonctionnement de l'estimateur. Le second registre renferme donc lui aussi cinq éléments qui sont les instants d'échantillonnage correspondants aux échantillons du premier registre. Le principe de fonctionnement de l'estimateur impose, pour des raisons qui seront expliquées par la suite, de tester non pas le produit $\mathrm{P}{ }_{k} \mathrm{P}{ }_{\mathrm{k}-1}$ mais le produit $\mathrm{P}{ }_{\mathrm{k}-2} \mathrm{P}{ }_{\mathrm{k}-3}$. L'équation (E.1) donnant le signal de commande s'écrit finalement :

$$
\mathrm{c}(\mathrm{k})=\operatorname{sgn}\left[1-\operatorname{sgn}\left(\mathrm{P}_{\mathrm{k}-3} \mathrm{P}^{*} \mathrm{k}-2\right)\right]
$$

\section{b) Estimateur des to:}

Au voisinage de zéro, on peut considérer que le signal sinusoïdal à l'entrée du détecteur de zéro varie linéairement avec le temps, c'est à dire :

$$
\mathrm{P}^{*}=\mathrm{at}+\mathrm{b}
$$

On procède alors à une interpolation afin de déterminer de manière précise l'instant "exact" du franchissement du zéro. Nous disposons donc, grâce au détecteur de zéro, d'un registre de cinq points d'échantillonnage $\mathrm{P}^{*}$ et d'un registre de temps. Pour mettre en oeuvre cette interpolation, on utilise la méthode des moindres carrés appliquée au cas simple d'une régression linéaire [9]. Afin, d'effectuer l'estimation des coefficients du modèle (E.3), il est nécessaire de posséder un certain nombre $\mathrm{N}$ de couples de mesure autour de ce passage par zéro. L'expérience nous a montré que $N=4$ ou 5 est un nombre suffisant pour une telle application. Comme il ne s'agit pas de faire de la prédiction, cela revient à écrire que les valeurs $\mathrm{P}^{*} \mathrm{k}$ et $\mathrm{P}^{*} \mathrm{k}-1$ sont nécessaires lorsqu'on détecte un zéro entre $\mathrm{t}_{\mathrm{k}-3}$ et $\mathrm{t}_{\mathrm{k}-2}$. Ceci explique donc la forme (E.2) du signal de commande à la sortie du détecteur de zéro. A la sortie de l'estimateur, nous disposons finalement des valeurs des estimations des coefficients a et $b$ de la relation

(E.3) que l'on note â et $\hat{b}$. Les instants "exacts" de passage par zéro sont donc donnés par :

$$
\hat{\mathbf{t}}_{0}=\hat{b} / \hat{a}
$$

c) Estimateur de fréquence:

Pour calculer la fréquence instantanée fondamentale du signal on applique la relation suivante :

$$
\hat{\mathrm{f}} 0^{\mathrm{i}}=\frac{1}{\hat{\mathrm{t}} 0^{\mathrm{i}}-\hat{\mathrm{t}} \mathbf{0}^{\mathrm{i}-2}}
$$

Le schéma complet et détaillé de l'estimateur de fréquence est alors:

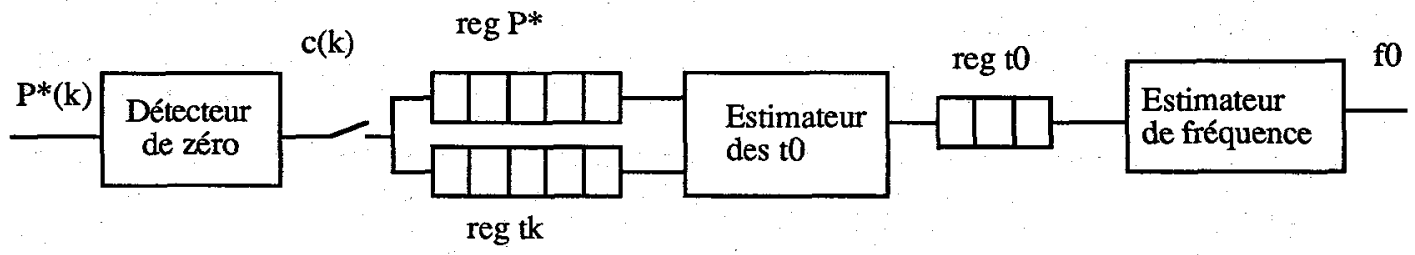

\section{RESULTATS}

Nous disposons d'un PC équipé d'une carte OROS AU22 et d'un logiciel de traitement de signal OPAL muni d'une bibliothèque d'applications sur TMS320C25. Le but que nous somme fixés est d'effectuer le traitement en temps réel grâce au logiciel OPAL, ce qui revient ici à effectuer l'estimation de $\hat{t}_{0}{ }^{i}$ avant le passage par zéro suivant de la sinusoïde. Ce traitement a donc été possible grâce à l'utilisation du logiciel OPAL. Cette solution a été testée sur deux configurations. Dans les deux cas la fréquence d'échantillonnage est fixe et égale à $3200 \mathrm{~Hz}$. Cette fréquence nous est apparue comme valeur limite pour un traitement en temps réel compte tenu du temps de calcul dû au filtrage et à la détermination de â et $\hat{b}$. 
A la sortie d'un générateur :

Le générateur de fréquence fournit à l'entrée du détecteur un signal carré de fréquence $f_{0}$. Le problème à résoudre ici est donc celui du temps de calcul. L'objectif que nous nous sommes fixé est la mise au point d'un système fonctionnant sur une plage de fréquence de $[25,100] \mathrm{Hz}$. De manière rigoureuse que nous considérons en fait deux plages de fréquences : $[25,50]$ et $[50,100] \mathrm{Hz}$. Chaque traitement fournit la valeur moyenne et la déviation standard. Les résultats ont montré que le dispositif est fiable comparé à la mesure effectuée à l'aide d'un fréquencemètre classique. Nous constatons une dispersion de $2 \%$ autour de la valeur mesurée sur celui-ci, et un écart inférieur à $0.2 \%$ après moyennage sur 20 périodes.

Sur le banc aéraulique :

Ce banc est constitué d'un ventilateur, d'un groupe pulsatoire faisant varier le débit massique de refoulement et d'une conduite cylindrique. Un capteur de pression a été placé sur la conduite afin de permettre l'acquisition du signal de pression à traiter.

Les résultats ont été comparés à ceux donnés par une FFT dont la résolution est de.2\%. Nous avons alors constatés que l'écart après moyennage sur 20 périodes est inférieur à $2 \%$. Ceci provient notamment du fait que le filtre n'annule pas en réalité entièrement les harmoniques.

\section{Remarque :}

Nous pouvons étendre l'application de ce type de détecteur à la détection de phase. En effet, il est facile de concevoir qu'une estimation de la phase absolue peut être obtenue directement à partir de l'estimation des $\hat{\mathrm{t}}_{\mathrm{O}}^{\mathrm{i}}$. La phase absolue s'écrit alors: $\phi=2 \pi \hat{\mathrm{f}}_{\mathrm{O}} \hat{\mathrm{t}}_{\mathrm{o}} \mathrm{i}$

Toutefois les essais ont montré que le cas de signaux à transitoires rapides.nécessite un développement supplémentaire.

\section{CONCLUSION}

Même si nous pouvons, suite à tous ces essais, considérer que nous avons validé le bon fonctionnement de ce type de détecteur de fréquence,un certain développement est encore nécessaire. En effet le calcul final de la fréquence tel qu'il est conçu, n'est pas directement exploitable sur la carte OROS. En conclusion le système developpé s'est montré fiable quant à ces résultats d'estimations mais demande d'être modifié, voire simplifié si nous voulons atteindre notre objectif premier qui reste le remplacement d'un codeur optique... Dans ce but nous avons supprimé le module de calcul d'interpolation. Cette modification nous a permis d'utiliser une sortie numérique sur laquelle est transféré un signal à la même fréquence que le fondamental qui nous sert de signal de synchronisation et un signal d'horloge à $\mathrm{Nf}_{\mathrm{o}}$ utilisé pour l'échantillonnage conditionnel. Nous avons testé et comparé ce nouveau dispositif à ceux d'un codeur optique : les premiers essais dans ce sens se sont révélés prometteurs.

Ce travail fait partie d'un ensemble de travaux subventionnés par le SRETIE du secrétariat d'état à l'environnement dans le cadre de l'appel d'offres " contrôle actif".

\section{Références:}

[1] S.J. ELLIOT-P.A.NELSON, The acive control of sound, Electronics \& Communication Engineering Journal, August 1990.

[2] J.PAILLARD, Multivariable digital controler architecture for noise control, Comptes-rendus des rencontres 'Contrôle actif vibro-acoustique et dynamique stochastique' Marseille, p 267-273, Octobre 1991.

[3] A.ROURE, Nouveaux développements du contrôle actif au LMA, Comptes-rendus des rencontres 'Contrôle actif vibro-acoustique et dynamique stochastique' Marseille, p 163-175, Octobre 1991.

[4] J.TARTARIN-J.LAUMONIER-J.L.PEUBE, Contrôle actif des pulsations de débit en écoulement, Comptes-rendus des rencontres 'Contrôle actif vibro-acoustique et dynamique stochastique' Marseille, p 213-225, Octobre 1991

[5] J.TARTARIN-J.LAUMONIER-J.L.PEUBE-M.DeCOLIGNY,Contrôle actif de débits instationnaires et atténuation de bruits d'écoulement, Premier Congrès Français d'acoustique, C2 1197-1200, Février 1990.

[6] G.S.GIILL-S.C.GUPTA, First order discrete phase locked loop with application to anglemodulated carrier, IEEE Trans. on Communications, p 454-462, June 1972.

[7] M.BELLANGER, Traitement numérique du signal, Masson, 1987.

[8] P.M.BEAUFILS-M.RAMI, Le filtrage numérique, Sybex, 1988.

[9] J.C.TRIGEASSOU, Recherche de modeles expérimentaux assistée par ordinateur, Technique et documentation Lavoisier, 1988. 